\title{
The width of Galton-Watson trees conditioned by the sizeit
}

\section{Michael Drmota and Bernhard Gittenberger}

Department of Discrete Mathematics and Geometry, Wiedner Hauptstr. 8-10/104, A-1040 Wien, Austria

received Apr 16, 2004, revised Aug 24, 2004, accepted Sep 2004.

It is proved that the moments of the width of Galton-Watson trees of size $n$ and with offspring variance $\sigma^{2}$ are asymptotically given by $(\sigma \sqrt{n})^{p} m_{p}$ where $m_{p}$ are the moments of the maximum of the local time of a standard scaled Brownian excursion. This is done by combining a weak limit theorem and a tightness estimate. The method is quite general and we state some further applications.

Keywords: Galton-Watson branching process, width, moment convergence theorem, Brownian excursion

\section{Introduction}

In this paper we are considering rooted trees which are family trees of a Galton-Watson branching process conditioned to have total progeny $n$. These trees are also called simply generated trees (see [35]). Without loss of generality we may assume that the offspring distribution $\xi$ is given by

$$
\mathbf{P}\{\xi=k\}=\frac{\tau^{k} \varphi_{k}}{\varphi(\tau)},
$$

where $\left(\varphi_{k} ; k \geq 0\right)$ is a sequence of non-negative numbers such that $\varphi_{0}>0$ and $\varphi(t)=\sum_{k \geq 0} \varphi_{k} t^{k}$ has a positive or infinite radius of convergence $R$ and $\tau$ is an arbitrary positive number within the circle of convergence of $\varphi(t)$. These conditions in particular imply that all moments of $\xi$ exist and that $\tau<R$. Due to conditioning on the total progeny and finiteness of moments it is no restriction if we confine ourselves to studying only the critical case, that is, $\mathbf{E} \xi=1$ which equivalently means that $\tau$ satisfies $\tau \varphi^{\prime}(\tau)=\varphi(\tau)$. The variance of $\xi$ can also be expressed in terms of $\varphi(t)$ and is given by

$$
\sigma^{2}=\frac{\tau^{2} \varphi^{\prime \prime}(\tau)}{\varphi(\tau)} .
$$

Note that the offspring distribution (1) can be interpreted as assigning weights to all trees defined by

$$
\omega(T)=\prod_{k \geq 0} \varphi_{k}^{n_{k}(T)}
$$

\footnotetext{
${ }^{\dagger}$ This research has been supported by the Austrian Science Foundation FWF, grant P16053.

1365-8050 @ 2004 Discrete Mathematics and Theoretical Computer Science (DMTCS), Nancy, France
} 
for a tree $T$ having $n$ nodes, $n_{k}$ of which have out-degree $k, k \geq 0$. Denote by $|T|$ the number of nodes of such a tree and let $a_{n}$ be the (weighted) number of all trees with $n$ nodes, i.e.

$$
a_{n}=\sum_{T:|T|=n} \omega(T)
$$

Then the corresponding generating function $a(z)=\sum_{n \geq 0} a_{n} z^{n}$ satisfies the functional equation

$$
a(z)=z \varphi(a(z)) .
$$

Denote by $\left(L_{n}(t), t \geq 0\right)$ the sequence of the generation sizes of a Galton-Watson tree the total progeny of which is $n$. For non-integer $t$ we define $L_{n}(t)$ by linear interpolation:

$$
L_{n}(t)=(\lfloor t\rfloor+1-t) L_{n}(\lfloor t\rfloor)+(t-\lfloor t\rfloor) L_{n}(\lfloor t\rfloor+1), \quad t \geq 0 .
$$

We are interested in the width of such a tree which is defined by

$$
w_{n}=\max _{t \geq 0} L_{n}(t) .
$$

This quantity attracted the interest of many authors. First, Odlyzko and Wilf [37] became interested in this tree parameter when studying the bandwidth

$$
\beta(T)=\min _{f}\left(\max _{(u, v) \in E(T)}|f(u)-f(v)|\right)
$$

of a tree $T$, where $f$ is an assignment of distinct integers to the vertices of the tree. They showed for a tree with $n$ vertices and height $h(T)$ and width $w(T)$ that

$$
\frac{n-1}{2 h(T)} \leq \beta(T) \leq 2 w(T)-1
$$

and furthermore they showed that there exist positive constants $c_{1}$ and $c_{2}$ such that the estimate

$$
c_{1} \sqrt{n}<\mathbf{E} w_{n}<c_{2} \sqrt{n \log n}
$$

holds. The exact order of magnitude was left as an open problem. Aldous conjectured [1, Conj. 4] that $L_{n}$ (suitably normalized) converges to Brownian excursion local time. This was first proved in [15], later by different methods by Kersting [29] and Pitman [38]. More precisely, set

$$
l_{n}(t)=\frac{2}{\sigma \sqrt{n}} L_{n}\left(\frac{2 t}{\sigma} \sqrt{n}\right)
$$

and

$$
l(t)=\lim _{\varepsilon \rightarrow 0} \frac{1}{\varepsilon} \int_{0}^{1} I_{[t, t+\varepsilon]}(W(s)) d s,
$$

where $(W(s), 0 \leq s \leq 1)$ is the standard scaled Brownian excursion. $l(t)$ is the local time (at time 1 and level $t$ ) of the normalized Brownian excursion. Then the above described limit theorem reads as follows: 
Theorem 1 ([15]). Let $\varphi(t)$ be the GF of a family of random trees. Assume that $\varphi(t)$ has a positive or infinite radius of convergence $R$. Furthermore suppose that the equation $t \varphi^{\prime}(t)=\varphi(t)$ has a minimal positive solution $\tau<R$. Then we have

$$
\left(l_{n}(t), t \geq 0\right) \stackrel{w}{\longrightarrow}(l(t), t \geq 0)
$$

in $C[0, \infty)$, as $n \rightarrow \infty$.

Partial results go back to [9, 22, 27, 34, 41]. The density of the finite dimensional distributions of $l$ was computed in [25]. A consequence of Theorem 1 is the following result which was proved directly by Takács [40].

Corollary 1 ([15]). Under the assumptions of Theorem 1$]$ we have

$$
\sup _{t \geq 0} l_{n}(t) \stackrel{w}{\longrightarrow} \sup _{t \geq 0} l(t)
$$

Thus this suggests (but does not imply) $\sqrt{n}$ as correct order of magnitude in (4).

Note that the maximum of local time is well studied (cf. [28, 8, 18, 3, 34]). We have

$$
\sup _{t \geq 0} l(t) \stackrel{d}{=} 2 \sup _{0 \leq t \leq 1} W(t)
$$

moreover it is theta-distributed, i.e.,

$$
\mathbf{P}\left\{\sup _{0 \leq t \leq 1} l(t) \leq x\right\}=1-2 \sum_{k \geq 1}\left(x^{2} k^{2}-1\right) e^{-x^{2} k^{2} / 2}, \quad x>0,
$$

and

$$
\mathbf{E}\left[\left(\sup _{t \geq 0} l(t)\right)^{p}\right]=2^{p / 2} p(p-1) \Gamma\left(\frac{p}{2}\right) \zeta(p) .
$$

The purpose of this paper is to show that, in addition to the weak limit theorem above, we have a moment convergence theorem of $\sup _{t \geq 0} l_{n}(t)$ to $\sup _{t \geq 0} l(t)$, too. We formulate it in terms of the width $w_{n}=\max _{t \geq 0} L_{n}(t)=(\sigma / 2) \sqrt{n} \sup _{t \geq 0} \bar{l}_{n}(t)$.

Theorem 2. Suppose that there exists a minimal positive solution $\tau<R$ of $t \varphi^{\prime}(t)=\varphi(t)$. Then the width $w_{n}$ satisfies

$$
\mathbf{E}\left(w_{n}^{p}\right)=\sigma^{p} 2^{-p / 2} p(p-1) \Gamma\left(\frac{p}{2}\right) \zeta(p) \cdot n^{p / 2} \cdot(1+o(1))
$$

as $n \rightarrow \infty$.

It should be further mentioned that Chassaing and Marckert [7] used the relation of parking functions and rooted trees as well as the strong convergence theorem of Komlos, Major and Tusnady [33] to derive tight bounds for the moments of the width for Cayley trees. They showed (here and throughout the whole paper, $a \ll b$ denotes $a \leq C b$ for some positive constant $C$ )

Theorem 3 ([7]). If $\varphi(t)=e^{t}$ and $p \geq 1$, then

$$
\left|\mathbf{E}\left(\frac{w_{n}}{\sigma \sqrt{n}}\right)^{p}-\mathbf{E}\left(\frac{1}{2} \sup _{t \geq 0} l(t)\right)^{p}\right|=\left|\mathbf{E}\left(\frac{w_{n}}{\sigma \sqrt{n}}\right)^{p}-\mathbf{E}\left(\sup _{t \geq 0} W(t)\right)^{p}\right| \ll n^{-p / 4} \log n .
$$


Remark. In fact, Chassaing and Marckert [7] showed an even stronger result: In some probability space there exist a sequence of copies of $w_{n}$ and a sequence of theta-distributed random variables $D_{n}$ such that for any $p \geq 1$

$$
\left\|\frac{2 w_{n}}{\sigma \sqrt{n}}-D_{n}\right\|_{p}=O\left(n^{-1 / 4} \sqrt{\log n}\right)
$$

where the $O$-constant depends on $p$.

Recently, Chassaing, Marckert, and Yor [6] have used Theorems 1 and 3 in conjunction with results of Aldous [1] to obtain a weak limit theorem (without moments) for the joint law of height and width of simply generated trees. (For binary trees they present an elementary proof, too.)

\section{Plan of the Proof of Theorem 2}

In view of Corollary 1 the result of Theorem 2 is not unexpected. Nevertheless, it does not follow directly from Corollary 1 since convergence of moments is not automatically transfered via weak convergence (from Theorem 1).

In order to prove Theorem 2 we actually use the result of Theorem 1 , that is, the normalized profile of Galton-Watson trees converges weakly to Brownian excursion local time: $\left(l_{n}(t), t \geq 0\right) \stackrel{w}{\longrightarrow}(l(t), t \geq 0)$. However, we need some additional considerations: In [17] (see also [14]) Drmota and Marckert introduced the notion of so-called polynomial convergence (that is inspired by the notion of uniform integrability). The key property for our purposes is the following one. It generalizes the results of [17] (see also [14, Theorem 3.7]) that only apply for processes with compact support.

Theorem 4. Let $x_{n}$ be a sequence of stochastic processes in $C[0, \infty)$ which converges weakly to $x$. Assume that for any choice of fixed positive integers $p$ and $d$ there exist positive constants $c_{0}, c_{1}, c_{2}, c_{3}$ such that

$$
\sup _{n \geq 0} \mathbf{E}\left|x_{n}(t)\right|^{p} \leq c_{0} e^{-c_{1} t} \text { for all } t \geq 0,
$$

and

$$
\sup _{n \geq 0} \mathbf{E}\left|x_{n}(t+s)-x_{n}(t)\right|^{2 d} \leq c_{2} e^{-c_{3} t} s^{d} \text { for all } s, t \geq 0 .
$$

Then $x_{n}$ is polynomially convergent to $x$, that is, for every continuous functional $F: C[0, \infty) \rightarrow \mathbb{R}$ of polynomial growth (i.e. $|F(y)| \ll\left(1+\|y\|_{\infty}\right)^{r}$ for some $\left.r \geq 0\right)$ we have

$$
\lim _{n \rightarrow \infty} \mathbf{E} F\left(x_{n}\right)=\mathbf{E} F(x) \text {. }
$$

We will show that $l_{n}$ satisfies the assumptions (5) and (6) of Theorem 4 and thus taking $F(x)=\|x\|_{\infty}^{r}$ yields immediately Theorem 2

The next section is devoted to the proof of Theorem 4 . In sections 4 and 5 we prove $(5)$ and $(6)$. Finally in section 6 we provide some further applications of Theorem 4.

\section{Proof of Theorem 4}

Let us start with the following two observations. 
Lemma 1. Suppose that $x_{n}$ satisfies (5). Then for every $p \geq 0$ we have

$$
\mathbf{E} \sup _{j \in \mathbb{N}}\left|x_{n}(j)\right|^{p} \ll 1
$$

uniformly for all $n$.

Proof. Since $\mathbf{E}\left|x_{n}(t)\right|^{p+1} \ll e^{-c_{1} t}$, uniformly in $n$, we have

$$
\begin{aligned}
\mathbf{P}\left\{\sup _{j \in \mathbf{N}}\left|x_{n}(j)\right| \geq A\right\} & \leq \sum_{j \geq 0} \mathbf{P}\left\{\left|x_{n}(j)\right| \geq A\right\} \\
& \leq \frac{1}{A^{p+1}} \sum_{j \geq 0} \mathbf{E}\left|x_{n}(j)\right|^{p+1} \text { by Markov's inequality } \\
& \ll \frac{1}{A^{p+1}} \sum_{j \geq 0} e^{-c_{1} j} \ll \frac{1}{A^{p+1}}
\end{aligned}
$$

Thus it follows that

$$
\mathbf{E}\left(\sup _{j \in \mathbf{N}}\left|x_{n}(j)\right|^{p}\right) \ll 1+p \int_{1}^{\infty} A^{p-1} \frac{1}{A^{p+1}} d A \ll 1 .
$$

Lemma 2. Suppose that $x_{n}$ satisfies (6). Then, for fixed $p$ we have

$$
\mathbf{E}\left(\sup _{|s-t| \leq \delta}\left|x_{n}(s)-x_{n}(t)\right|^{p}\right) \ll \delta^{p / 2} .
$$

uniformly for $\delta$ with $0<\delta<1$ and for all $n$.

Proof. First we prove that for every integer $d>1$ there exists a constant $K>0$ such that for $\varepsilon>0$ and $0<\delta<1$

$$
\mathbf{P}\left\{\sup _{|s-t| \leq \delta}\left|x_{n}(s)-x_{n}(t)\right| \geq \varepsilon\right\} \leq K \frac{\delta^{d-1}}{\varepsilon^{2 d}} .
$$

Arguing as in [4, pp. 95] guarantees that there exists a constant $K_{1}>0$ such that for all $m \geq 0$

$$
\mathbf{P}\left\{\sup _{|s-t| \leq \delta, m \leq s, t \leq m+2}\left|x_{n}(s)-x_{n}(t)\right| \geq \varepsilon\right\} \leq K_{1} e^{-c_{5} m} \frac{\delta^{d-1}}{\varepsilon^{2 d}} .
$$

Thus

$$
\mathbf{P}\left\{\sup _{|s-t| \leq \delta}\left|x_{n}(s)-x_{n}(t)\right| \geq \varepsilon\right\} \leq \sum_{m=0}^{\infty} K_{1} e^{-c_{5} m} \frac{\delta^{d-1}}{\varepsilon^{2 d}} \leq K \frac{\delta^{d-1}}{\varepsilon^{2 d}}
$$

for some constant $K>0$. 
Set

$$
Z=\sup _{|s-t| \leq \delta}\left|x_{n}(s)-x_{n}(t)\right| .
$$

Then by applying (7) it follows that (if $2 d \geq p+1$ )

$$
\begin{aligned}
\mathbf{E} Z^{p} & =p \int_{0}^{\infty} z^{p-1} \mathbf{P}[Z>z] d z \\
& =p \int_{0}^{(K \delta)^{(d-1) / d}} z^{p-1} \mathbf{P}[Z>z] d z+p \int_{(K \delta)^{(d-1) / d}}^{\infty} z^{p-1} \mathbf{P}[Z>z] d z \\
& \leq(K \delta)^{p(d-1) / d}+p K \delta^{d-1} \int_{(K \delta)^{(d-1) / d}}^{\infty} z^{p-1-2 d} d z \\
& \ll \delta^{p(d-1) / d} \leq \delta^{p / 2},
\end{aligned}
$$

which proves the Lemma.

The proof of Theorem 4 is now an easy task. Note that the results of Lemma 1 and 2 in conjunction with the triangular inequality imply

$$
\sup _{n \geq 0} \mathbf{E}\left(\sup _{t \geq 0}\left|x_{n}(t)\right|^{r}\right)<\infty \text { for all } r \geq 0 .
$$

Thus, if $F$ is a continuous functional of polynomial growth we have for any $\varepsilon>0$

$$
\sup _{n \geq 0} \mathbf{E}\left|F\left(x_{n}\right)\right|^{1+\varepsilon}<\infty
$$

By continuity of $F$ we also obtain $F\left(x_{n}\right) \stackrel{w}{\longrightarrow} F(x)$ and finally, by Billingsley [5, p. 338] it directly follows that

$$
\lim _{n \rightarrow \infty} \mathbf{E} F\left(x_{n}\right)=\mathbf{E} F(x)
$$

as desired.

\section{Moments for the Profile of Galton-Watson Trees}

We start with a lemma on the growth of coefficients of powers of certain generating functions.

Lemma 3. Let $z_{0} \neq 0$ and $\Delta=\left\{z:|z|<z_{0}+\eta,\left|\arg \left(z-z_{0}\right)\right|>\vartheta\right\}$, where $\eta>0$ and $0<\vartheta<\pi / 2$. Suppose that $f(z)$ and $g(z)$ are analytic functions in $\Delta$ which satisfy

$$
\begin{aligned}
& |f(z)| \leq \exp \left(-C \sqrt{\left|1-\frac{z}{z_{0}}\right|}\right), \quad z \in \Delta, \\
& g(z)=1-D \sqrt{1-\frac{z}{z_{0}}}+O\left(\left|1-\frac{z}{z_{0}}\right|\right), \quad z \in \Delta,
\end{aligned}
$$


for some positive constants $C, D$. Then for any fixed $\ell$ there exists a constant $C^{\prime}>0$ such that

$$
\left[z^{n}\right] \frac{f(z)^{r}}{(1-g(z))^{\ell}}=O\left(e^{-C^{\prime} r / \sqrt{n}} n^{(\ell-2) / 2}\right)
$$

uniformly for all $r, n \geq 0$ (where $\left[z^{n}\right] F(z)$ denotes the coefficient of $z^{n}$ of the function $F(z)$ ).

Proof. The only difference to [23, Lemma 3.5] is the factor $1 /(1-g(z))^{\ell}$, but since its behavior in $\Delta$ is known and [20, Theorem 3] is applicable, the proof is analogous to that of [23, Lemma 3.5].

By means of this lemma we can show

Lemma 4. For every fixed integer $p>0$ there exist positive constants $c_{0}$ and $c_{1}$ such that

$$
\sup _{n \geq 0} \mathbf{E} l_{n}(t)^{p} \leq c_{0} e^{-c_{1} t}
$$

for all $t \geq 0$.

Proof. For technical simplicity we assume that $g=\operatorname{gcd}\left\{i \geq 1: \varphi_{i}>0\right\}=1$. This assumption ensures that the tree function $a(z)$ defined by $\left(3\right.$ has only one singularity $z_{0}=1 / \varphi^{\prime}(\tau)$ on the circle of convergence. If $g=\operatorname{gcd}\left\{i \geq 0: \varphi_{i}>0\right\}>1$ then we can use the substitution $x=z^{1 / g}$ to get $a(z)=x b(x)$ where $b(x)$ is analytic with only one singularity on the circle of convergence. Thus this case reduces to the case $g=1$. The other possibility is to deal with the $g$ singularities $z_{0} e^{2 \pi i j / g}, j=0,1, \ldots, g-1$, on the circle of convergence and add all contributions.

In particular, it is also well known that (if $g=1) a(z)$ admits a representation of the following kind

$$
a(z)=\tau-\frac{\tau \sqrt{2}}{\sigma} \sqrt{1-\frac{z}{z_{0}}}+O\left(\left|1-\frac{z}{z_{0}}\right|\right)
$$

that is valid for $|z|<z_{0}+\eta$ and $\arg \left(z-z_{0}\right) \neq 0$, where $\eta>0$ is suitably small, compare with [35] and [13].

In what follows we will need the local expansion of $\alpha(z)=z \varphi^{\prime}(a(z))$. From 96 we immediately get

$$
\alpha(z)=1-\sigma \sqrt{2} \sqrt{1-\frac{z}{z_{0}}}+O\left(\left|1-\frac{z}{z_{0}}\right|\right)
$$

for $|z|<z_{0}+\eta$ and $\arg \left(z-z_{0}\right) \neq 0$.

Due to $10 \mid$ there exists a constant $C>0$ such that $|\alpha(z)| \leq \exp \left(-C \sqrt{\left|1-z / z_{0}\right|}\right)$ for $z \in \Delta$ (with $\Delta$ from Lemma 3). Furthermore, it follows that

$$
\sup _{z \in \Delta}|\alpha(z)|=1,
$$

where we have to choose $\eta>0$ and $0<\vartheta<\pi / 2$ in a proper way. First, since the power series of $\alpha(z)$ has only positive coefficients, we have $\max _{|z| \leq z_{0}}|\alpha(z)|=1$. If we assume that $d=\operatorname{gcd}\left\{i \geq 1: \varphi_{i}>0\right\}=1$ it also follows that

$$
\max _{|z| \leq z_{0},\left|z-z_{0}\right| \geq \varepsilon}|\alpha(z)|<1
$$


for every $\varepsilon>0$. Now, in the vicinity of the singularity $z_{0}$, that is, for $\left|z-z_{0}\right|<\varepsilon$ we can again use 10] and get for $z=z_{0}\left(1+t e^{i \theta}\right)$

$$
\left|\alpha\left(1+t e^{i \theta}\right)\right|=\left|1-\sigma \sqrt{2 t} e^{ \pm i(\pi-\theta) / 2}+O(t)\right|
$$

where $\theta>\pi / 2$. Hence we have $|\alpha(z)| \leq 1$ for $\left|z-z_{0}\right| \leq \varepsilon$ and $\left|\arg \left(z-z_{0}\right)\right|>\theta$. Finally, for $|z| \leq z_{0}+\eta$ and $\left|z-z_{0}\right| \geq \varepsilon$ we obtain the same inequality from $[12)$ by a continuity argument (for some sufficiently small $\eta>0$ ). This proves 11 .

Now observe that by substituting $r=\lfloor t \sqrt{n}\rfloor$ in $(8)$ we get

$$
\mathbf{E} L_{n}(r)^{p} \leq c_{0} e^{-c_{1} r / \sqrt{n}} n^{p / 2} .
$$

Furthermore note that it suffices to show 13 for the $p$ th factorial moment

$$
\mathbf{E}\left[L_{n}(r)\right]_{p}=\mathbf{E} L_{n}(r)\left(L_{n}(r)-1\right) \cdots\left(L_{n}(r)-p+1\right)
$$

instead of the $p$ th moment, which we can easily express in terms of the proper coefficient of a generating function. Indeed we have

$$
\mathbf{E}\left[L_{n}(r)\right]_{p}=\left.\frac{1}{a_{n}}\left[z^{n}\right]\left(\frac{\partial}{\partial u}\right)^{p} y_{r}(z, u a(z))\right|_{u=a(z)},
$$

where

$$
\begin{aligned}
y_{0}(z, u) & =u \\
y_{i+1}(z, u) & =z \varphi\left(y_{i}(z, u)\right), \quad i \geq 0 .
\end{aligned}
$$

In order to evaluate this coefficient we use Lemma 3 which translates the local behavior of the function near its singularity into an asymptotic estimate for the coefficients.

By [24, p. 287, equ. (22)] we have

$$
\left.\left(\frac{\partial}{\partial u}\right)^{p} y_{r}(z, u a(z))\right|_{u=a(z)}=O\left(a(z)^{p}\left|\alpha(z)^{r}\right|\left|\frac{1-\alpha(z)^{r}}{1-\alpha(z)}\right|^{p-1}\right) .
$$

From 11 we get

$$
\max _{z \in \Delta}\left|\frac{1-\alpha(z)^{r}}{1-\alpha(z)}\right| \leq r
$$

Moreover $a(z)^{p}$ behaves like a constant near the singularity and $\alpha(z)^{r}$ meets the condition in Lemma 3 . Hence the last factor in 15 is bounded by $r^{p-1}$ and hence contributes a factor $n^{(p-1) / 2}$ to the order of magnitude of the $p$ th factorial moment $\mathbf{E}\left[L_{n}(r)\right]_{p}$. Applying Lemma 3 , which yields $\exp \left(-c_{1} r / \sqrt{n}\right)$, and normalizing by $a_{n} \sim \tau / \sigma z_{0}^{n} \sqrt{2 \pi n^{3}}$ we get the desired result. 


\section{Quantitative Tightness Estimates}

With help of Lemma 3 we can prove the following quantitative tightness estimate.

Lemma 5. For every fixed positive integer $d$ there exist constants $c_{2}, c_{3}$ such that for every $s, t>0$

$$
\mathbf{E}\left|l_{n}(t+s)-l_{n}(t)\right|^{2 d} \leq c_{2} e^{-c_{3} t} s^{d} .
$$

Proof(Sketch). Observe that we can rewrite (17) as

$$
\mathbf{E}\left|L_{n}(r)-L_{n}(r+h)\right|^{2 d} \leq c_{2} e^{-c_{3} r / \sqrt{n}} h^{d} n^{d / 2}
$$

which is quite similar to [15, Theorem 6.1]. From [15] it follows that

$$
\mathbf{E}\left|L_{n}(r)-L_{n}(r+h)\right|^{2 d}=\frac{1}{a_{n}}\left[z^{n}\right] H_{r, h}(z),
$$

in which

$$
H_{r, h}(z)=\left.\left(u \frac{\partial}{\partial u}\right)^{2 d} y_{r}\left(z, u y_{h}\left(z, u^{-1} a(z)\right)\right)\right|_{u=1}
$$

and $y(z, u)$ is given by $(14)$.

Evaluation of this coefficient is again done by Lemma 3. By [15, Proposition 6.1] it is easy to show that

$$
H_{r, h}(z)=\alpha(z)^{r} \sum_{j=0}^{d} G_{j, r h}(z) \frac{\left(1-\alpha(z)^{h}\right)^{j}}{(1-\alpha(z))^{d-1+j}}
$$

where $G_{j, r h}(z)$ satisfy

$$
\max _{z \in \Delta}\left|G_{j, r h}(z)\right|=O(1) .
$$

Eventually, an application of (16), with $h$ instead of $r$, and Lemma 3 to (19) yields

$$
\left[z^{n}\right] H_{r, h}(z)=O\left(\frac{h^{d} n^{(d-3) / 2}}{z_{0}^{n}}\right)
$$

and, thus, by $a_{n} \sim \tau / \sigma z_{0}^{n} \sqrt{2 \pi n^{3}}$ the proof is complete.

\section{Extensions}

\subsection{Nodes of given degree}

In [12] the number of nodes with fixed degree $d$ in layers of random trees was investigated. In this case also limit theorems like Theorem 1 and Corollary 1 hold. In fact, we have

Theorem 5. Let $L_{n}^{(d)}(k)$ denote the number of nodes with degree $d$ in layer $k$ in a random tree of total progeny $n$. Furthermore, set for any $t \geq 0$

$$
l_{n}^{(d)}(t)=\frac{2}{\sigma c_{d} \sqrt{n}} L_{n}^{(d)}\left(\frac{2 t}{\sigma} \sqrt{n}\right)
$$

where $c_{d}=\varphi_{d-1} \tau^{d-1} / \varphi(\tau)$. Then we have 
1.

$$
l_{n}^{(d)} \stackrel{w}{\longrightarrow} l \text { and } \sup _{t \geq 0} l_{n}^{(d)}(t) \stackrel{w}{\longrightarrow} \sup _{t \geq 0} l(t)
$$

2.

$$
\mathbf{E}\left(\left(w_{n}^{(d)}\right)^{p}\right)=\mathbf{E}\left(\sup _{t \geq 0} l(t)\right)^{p}(1+o(1)),
$$

where $w_{n}^{(d)}=\max _{k \geq 0} l_{n}^{(d)}(k)$.

Proof(Sketch).

Part 1 was proved in [12]. The proof of part 2 runs similarly to the proof of Theorem 2 The only crucial point is to get estimates as in Lemma 4 and Lemma 5 , namely

$$
\mathbf{E} L_{n}^{(d)}(r)^{p} \leq c_{1} e^{-c_{2} r / \sqrt{n}} n^{p / 2}
$$

and

$$
\mathbf{E}\left|L_{n}^{(d)}(r)-L_{n}^{(d)}(r+h)\right|^{2 d} \leq c_{1} e^{-c_{2} r / \sqrt{n}} h^{d} n^{d / 2} .
$$

Both inequalities can be proved in a similar manner, so let us look at the second one (the first is the easier one). The results in [12] imply

$$
\mathbf{E}\left|L_{n}^{(d)}(r)-L_{n}^{(d)}(r+h)\right|^{2 d}=\frac{2}{\sigma a_{n}}\left[z^{n}\right] H_{2 r / \sigma, 2 h / \sigma}^{(d)}(z)
$$

with

$$
\begin{aligned}
H_{r, h}^{(d)}(z)= & \left(u \frac{\partial}{\partial u}\right)^{2 d} y_{r}\left(z, z(u-1) \varphi_{d-1} y_{h-1}\left(z, z\left(u^{-1}-1\right) \varphi_{d-1} a(z)^{d-1}+a(z)\right)\right. \\
& \left.y_{h}\left(z, z\left(u^{-1}-1\right) \varphi_{d-1} a(z)^{d-1}+a(z)\right)\right)\left.\right|_{u=1}
\end{aligned}
$$

and since the right-hand side of this equation can be expressed in a form similar to [19], we can easily prove 20 .

\subsection{Strata of random mappings}

A random mapping of size $n$ is an element of the set $F_{n}$ of all mappings of a set with $n$ elements into itself, where $F_{n}$ is equipped with the uniform distribution. These mappings can be represented by functional digraphs consisting of components which are cycles of trees, i.e., each component of this graph contains exactly one cycle and each vertex in this cycle is the root of a tree in which each edge is directed towards the root.

The set of points in distance $r$ from a cycle is called the $r$ th stratum of a random mapping. This parameter was previously studied in [2, 11, 16, 36, 39]. For general results on random mappings and literature see [32, 21]. Let $M_{n}(r)$ denote the number of nodes in the $r$ th stratum of a random mapping of size $n$. Then in [16] we proved 
Theorem 6. Let $B(t)$ denote reflecting Brownian bridge, i.e., a process on the interval $[0,1]$ which is identical in law to $|\bar{W}(s)-s \bar{W}(1)|\left(\bar{W}(t)\right.$ is the standard Brownian motion), and $l^{(B)}(t)$ its local time, i.e.,

$$
l^{(B)}(t)=\lim _{\varepsilon \rightarrow 0} \frac{1}{\varepsilon} \int_{0}^{1} I_{[t, t+\varepsilon]}(B(s)) d s .
$$

Then we have

$$
\left(m_{n}(t), t \geq 0\right)=\left(\frac{2}{\sqrt{n}} M_{n}(2 t \sqrt{n}), t \geq 0\right) \stackrel{w}{\longrightarrow}\left(l^{(B)}(t), t \geq 0\right)
$$

in $C[0, \infty)$, as $n \rightarrow \infty$. Thus we also have

$$
\sup _{t \geq 0} m_{n}(t) \stackrel{w}{\longrightarrow} \sup _{t \geq 0} l^{(B)}(t) .
$$

Here again the corresponding moment convergence theorem is not a consequence of 21. However, as before we can show

Theorem 7. We have

$$
\mathbf{E}\left(\left(\sup _{t \geq 0} m_{n}(t)\right)^{p}\right)=\mathbf{E}\left(\sup _{t \geq 0} l^{(B)}(t)\right)^{p}(1+o(1))
$$

Proof(Sketch). Again the crucial point is to get proper estimates. From [16] it is an easy exercise to get

$$
\mathbf{E}\left|M_{n}(r)-M_{n}(r+h)\right|^{2 d}=\frac{2 n !}{n^{n}}\left[z^{n}\right] H_{2 r, 2 h}(z),
$$

in which

$$
H_{r, h}(z)=\left.\left(u \frac{\partial}{\partial u}\right)^{2 d} \frac{1}{1-y_{r}\left(z, u y_{h}\left(z, u^{-1} a(z)\right)\right)}\right|_{u=1} .
$$

This function can be written in a form similar to 19$]$ and thus we can easily prove

$$
\mathbf{E}\left|M_{n}(r)-M_{n}(r+h)\right|^{2 d} \leq c_{1} e^{-c_{2} r / \sqrt{n}} h^{d} n^{d / 2}
$$

and then 22]. The corresponding bound for the moments, obtained in the same way, carries out even easier.

\subsection{Height of random trees}

The same method can be used to re-derive the analogue for the height $h_{n}$ of simply generated trees (see Flajolet and Odlyzko [19]).

Theorem 8. Suppose that there exists a minimal positive solution $\tau<R$ of $t \varphi^{\prime}(t)=\varphi(t)$. Then

$$
\mathbf{E}\left(h_{n}^{p}\right)=\left(\frac{\sqrt{2 n}}{\sigma}\right)^{p} p(p-1) \Gamma\left(\frac{p}{2}\right) \zeta(p)(1+o(1))
$$

as $n \rightarrow \infty$. 
$h_{n}$ is equal to the maximum of the traversal process $T_{n}(r)$, defined to be the distance between the root and the $r$ th node during preorder traversal of the tree. Obviously, the same holds when we only traverse leaves (call the corresponding process $\hat{T}_{n}(r)$ ). It is well known (see [1]) that

$$
\left(X_{n}(t), t \geq 0\right)=\left(\frac{1}{\sqrt{n}} T_{n}(2 t n) t \geq 0\right) \stackrel{w}{\longrightarrow}\left(\frac{2}{\sigma} W(t) t \geq 0\right)
$$

The height of leaves was investigated by several authors (see [30, 31, 26, 10, 23]. Here a similar limit theorem holds: With $\hat{X}_{n}(t)=\hat{T}_{n}(t n) / \sqrt{n}$ we have (see [23])

$$
\left(\hat{X}_{n}\left(\frac{\varphi_{0}}{\varphi(\tau)} t\right) t \geq 0\right) \stackrel{w}{\longrightarrow}\left(\frac{2}{\sigma} W(t) t \geq 0\right) .
$$

In addition, in [23] the tightness estimate

$$
P\left\{\left|\hat{X}_{n}(s)-\hat{X}_{n}(t)\right| \geq \varepsilon\right\} \leq C \frac{1}{\varepsilon^{4}|s-t|} \exp \left(-D \frac{\varepsilon}{\sqrt{|s-t|}}\right)
$$

for some positive constants $C$ and $D$ was shown. This can be used to derive moment estimates like in Lemma 5 and then one proceeds as in the previous section to re-derive Flajolet and Odlyzko's [19] result on the moments of the height.

Finally, we want to mention that it is also possible to obtain the moments of the height of a random mapping (this was done by Flajolet and Odlyzko [21]) by our method. One has to use the weak limit theorem by Aldous and Pitman [2] and derive a tightness estimate in a similar fashion as has been done in [16].

\section{References}

[1] David Aldous. The continuum random tree. II. An overview. In Stochastic analysis (Durham, 1990), volume 167 of London Math. Soc. Lecture Note Ser., pages 23-70. Cambridge Univ. Press, Cambridge, 1991.

[2] David J. Aldous and Jim Pitman. Brownian bridge asymptotics for random mappings. Random Structures Algorithms, 5(4):487-512, 1994.

[3] $\mathrm{Ph}$. Biane and M. Yor. Valeurs principales associées aux temps locaux browniens. Bull. Sci. Math. (2), 111(1):23-101, 1987.

[4] Patrick Billingsley. Convergence of probability measures. John Wiley \& Sons Inc., New York, 1968.

[5] Patrick Billingsley. Probability and measure. Wiley Series in Probability and Mathematical Statistics. John Wiley \& Sons Inc., New York, 1995.

[6] P. Chassaing, J. F. Marckert, and M. Yor. The height and width of simple trees. In Mathematics and computer science (Versailles, 2000), Trends Math., pages 17-30. Birkhäuser, Basel, 2000.

[7] Philippe Chassaing and Jean-François Marckert. Parking functions, empirical processes, and the width of rooted labeled trees. Electron. J. Combin., 8(1):Research Paper 14, 19 pp. (electronic), 2001. 
[8] Kai Lai Chung. Excursions in Brownian motion. Ark. Mat., 14(2):155-177, 1976.

[9] J. W. Cohen and G. Hooghiemstra. Brownian excursion, the $M / M / 1$ queue and their occupation times. Math. Oper. Res., 6(4):608-629, 1981.

[10] Michael Drmota. Distribution of the height of leaves of rooted trees. Diskret. Mat., 6(1):67-82, 1994.

[11] Michael Drmota. Correlations on the strata of a random mapping. Random Structures Algorithms, 6(2-3):357-365, 1995.

[12] Michael Drmota. On nodes of given degree in random trees. In Probabilistic methods in discrete mathematics (Petrozavodsk, 1996), pages 31-44. VSP, Utrecht, 1997.

[13] Michael Drmota. Systems of functional equations. Random Structures Algorithms, 10(1-2):103124, 1997.

[14] Michael Drmota. Stochastic analysis of tree-like data structures. Proc. R. Soc. Lond. Ser. A Math. Phys. Eng. Sci., 460(2041):271-307, 2004.

[15] Michael Drmota and Bernhard Gittenberger. On the profile of random trees. Random Structures Algorithms, 10(4):421-451, 1997.

[16] Michael Drmota and Bernhard Gittenberger. Strata of random mappings-a combinatorial approach. Stochastic Process. Appl., 82(2):157-171, 1999.

[17] Michael Drmota and Jean-François Marckert. Reinforced weak convergence of stochastic processes. Statistics Probab. Letters. To appear.

[18] Richard T. Durrett, Donald L. Iglehart, and Douglas R. Miller. Weak convergence to Brownian meander and Brownian excursion. Ann. Probability, 5(1):117-129, 1977.

[19] Philippe Flajolet and Andrew Odlyzko. The average height of binary trees and other simple trees. $J$. Comput. System Sci., 25(2):171-213, 1982.

[20] Philippe Flajolet and Andrew Odlyzko. Singularity analysis of generating functions. SIAM J. Discrete Math., 3(2):216-240, 1990.

[21] Philippe Flajolet and Andrew M. Odlyzko. Random mapping statistics. In Advances in cryptologyEUROCRYPT '89 (Houthalen, 1989), volume 434 of Lecture Notes in Comput. Sci., pages 329-354. Springer, Berlin, 1990.

[22] R. K. Getoor and M. J. Sharpe. Excursions of Brownian motion and Bessel processes. Z. Wahrsch. Verw. Gebiete, 47(1):83-106, 1979.

[23] Bernhard Gittenberger. On the contour of random trees. SIAM J. Discrete Math., 12(4):434-458 (electronic), 1999.

[24] Bernhard Gittenberger. On the profile of random forests. In Mathematics and computer science, II (Versailles, 2002), Trends Math., pages 279-293. Birkhäuser, Basel, 2002. 
[25] Bernhard Gittenberger and Guy Louchard. The Brownian excursion multi-dimensional local time density. J. Appl. Probab., 36(2):350-373, 1999.

[26] W. Gutjahr and G. Ch. Pflug. The asymptotic contour process of a binary tree is a Brownian excursion. Stochastic Process. Appl., 41(1):69-89, 1992.

[27] G. Hooghiemstra. On the explicit form of the density of Brownian excursion local time. Proc. Amer. Math. Soc., 84(1):127-130, 1982.

[28] Douglas P. Kennedy. The distribution of the maximum Brownian excursion. J. Appl. Probability, 13(2):371-376, 1976.

[29] Götz Kersting. On the height profile of a conditioned galton-watson tree. 1998. Unpublished.

[30] Peter Kirschenhofer. On the height of leaves in binary trees. J. Combin. Inform. System Sci., 8(1):4460, 1983.

[31] Peter Kirschenhofer. Some new results on the average height of binary trees. Ars Combin., 16(A):255-260, 1983.

[32] Valentin F. Kolchin. Random mappings. Translation Series in Mathematics and Engineering. Optimization Software Inc. Publications Division, New York, 1986.

[33] J. Komlós, P. Major, and G. Tusnády. An approximation of partial sums of independent RV's, and the sample DF. II. Z. Wahrscheinlichkeitstheorie und Verw. Gebiete, 34(1):33-58, 1976.

[34] G. Louchard. Kac's formula, Levy's local time and Brownian excursion. J. Appl. Probab., 21(3):479-499, 1984.

[35] A. Meir and J. W. Moon. On the altitude of nodes in random trees. Canad. J. Math., 30(5):997-1015, 1978.

[36] Ljuben R. Mutafchiev. The limit distribution of the number of nodes in low strata of a random mapping. Statist. Probab. Lett., 7(3):247-251, 1988.

[37] Andrew M. Odlyzko and Herbert S. Wilf. Bandwidths and profiles of trees. J. Combin. Theory Ser. $B, 42(3): 348-370,1987$.

[38] Jim Pitman. The SDE solved by local times of a Brownian excursion or bridge derived from the height profile of a random tree or forest. Ann. Probab., 27(1):261-283, 1999.

[39] G. V. Proskurin. The distribution of the number of vertices in the strata of a random mapping. Teor. Verojatnost. i Primenen., 18:846-852, 1973.

[40] Lajos Takács. Limit distributions for queues and random rooted trees. J. Appl. Math. Stochastic Anal., 6(3):189-216, 1993.

[41] Lajos Takács. Brownian local times. J. Appl. Math. Stochastic Anal., 8(3):209-232, 1995. 